\title{
Direct Ellipsometry Problem for the Substrate-Based Uniaxial Non-Homogeneous Film
}

\author{
M.M. KARPUK ${ }^{a, *}$ AND S.M. KARPUK ${ }^{b}$ \\ ${ }^{a}$ Koszalin Technical University \\ al. Kwiatkowskiego 6-E, 75-343 Koszalin, Poland \\ ${ }^{b}$ Department of Laser Physics and Spectroscopy, Belarussian State University \\ Fr. Skaryna ave. 4, 220050 Minsk, Belarus
}

(Received February 1, 2001; revised version September 27, 2001)

\begin{abstract}
An iterative procedure of calculation of reflectivities and transmission coefficients of light rays for an uniaxial film with a lapse rate of an index of refraction on a uniaxial substrate was gained by orienting the axis of anisotropy along the normal to the boundary. With its help the dependences of ellipsometric angles $\Delta$ and $\psi$ for a linear, quadratic, and sine-shaped profiles of refractive indices of a uniaxial immersing film with optic axis oriented along the normal to the boundary were analyzed. The dependences of angles $\Delta$ and $\psi$ on quantity of an uptake and anisotropy of the film were also examined. The numerical modelling for $\mathrm{ZnO}$ films and Langmuir-Blodgett-like films on a melted quartz was carried out, and this allowed to draw conclusions of practical importance for the ellipsometric investigations of the film structures.
\end{abstract}

PACS numbers: $78.20 .-\mathrm{e}, 44.30 .+\mathrm{v}$

\section{Introduction}

The modern technology of production of thin-film materials for microelectronics and integrated optics sets great demands to the properties of created structures and methods of controlling them [1]. One of perspective methods of controlling is ellipsometry [2,3], providing great accuracy of measurements and allowing to held investigations of film systems during their production (in situ) [3]. Nowadays anisotropic films, widely used in different optical and acoustoelectronic [4]

*corresponding author; e-mail: mikarpuk@hotmail.com 
devices, are of great interest. For effective control of anisotropic layered structures the knowledge of dependences of ellipsometric angles $\Delta$ and $\psi$ on parameters of the film and substrate, such as complex indices of refraction $N_{\mathrm{o}}, N_{\mathrm{e}}$, the angles of orientation of optical axes $\varphi, \Theta$ to boundary are required. Thus parameters of the film and the substrates in the main case are functions of coordinates. In particular, film refractive indices and orientation of optical axis depend on the thickness of the film.

The purpose of the given paper is the development of algorithms for finding the ellipsometric angles of uniaxial immersing films for arbitrary gradients of refractive indices, stating the dependences between them for different thicknesses of films and the analysis of opportunities of definition of refractive index gradients on ellipsometric measurements.

\section{Theory}

When modelling reflection and transmission of a plane electromagnetic wave on a boundary of media with gradients of refractive index, the method of dividing of a non-homogeneous medium into homogeneous flat layers is used. Let us consider some concepts at reflection of light on a plane boundary of semi-infinite anisotropic media and at reflection from an anisotropic layer.

In the ellipsometry of anisotropic media reflection and refraction of light on the plane boundary of semi-infinite media can be characterized by matrices of reflection $R$ and transmission $T$. Let us imagine that an electromagnetic wave having two orthogonally related components of complex amplitudes $\left(A_{\mathrm{p}}, A_{\mathrm{s}}\right)$ (Fig. 1a) impinges on a plane boundary isotropic medium-uniaxial crystal with complex refractive indices $N_{10}=N_{1 \mathrm{e}}=N_{1}$ and $N_{20}, N_{2 \mathrm{e}}$, respectively, under angle $\alpha$. Component $A_{\mathrm{p}}$ is situated on the plane of incidence of the wave, and component $A_{\mathrm{s}}$ is perpendicular to the planes of incidence. In a uniaxial crystal there arise ordinary and extraordinary waves with amplitudes $A_{\mathrm{o}}, A_{\mathrm{e}}$ respectively, that have in the general case arbitrary orientation concerning the plane of incidence. The field of reflected $\left(A_{\mathrm{p}}^{\prime}, A_{\mathrm{s}}^{\prime}\right)$ and past $\left(A_{\mathrm{e}}, A_{\mathrm{o}}\right)$ waves at arbitrary orientation of optical axes of the film and of the substrate are featured by $2 \times 2$ matrices of reflection and transmission [2]:

$$
\left(\begin{array}{c}
A_{\mathrm{p}}^{\prime} \\
A_{\mathrm{s}}^{\prime}
\end{array}\right)=R\left(\begin{array}{c}
A_{\mathrm{p}} \\
A_{\mathrm{s}}
\end{array}\right), \quad\left(\begin{array}{c}
A_{\mathrm{e}} \\
A_{\mathrm{o}}
\end{array}\right)=T\left(\begin{array}{c}
A_{\mathrm{p}} \\
A_{\mathrm{s}}
\end{array}\right),
$$

where

$$
R=\left(\begin{array}{cc}
R_{\mathrm{pp}} & R_{\mathrm{ps}} \\
R_{\mathrm{sp}} & R_{\mathrm{ss}}
\end{array}\right), \quad T=\left(\begin{array}{ll}
T_{\mathrm{ep}} & T_{\mathrm{es}} \\
T_{\mathrm{op}} & T_{\mathrm{os}}
\end{array}\right) .
$$

The diagonal elements $R_{\mathrm{pp} \text { ss }}$ of reflection matrix $R$ characterize the transformation of p- and s-polarized radiation of the same type, and non-diagonal $R_{\mathrm{ps} \text { sp }}$ - the transformation of s-polarized wave to p-polarized and back. For the transmission 
matrix $T$ elements $T_{\mathrm{ep}}, T_{\mathrm{es}}$ characterize the transformation of p- and s-polarized waves to extraordinary, and elements $T_{\mathrm{op}}, T_{\mathrm{os}}$ characterize the transformation of p- and s-polarized waves to an ordinary wave.
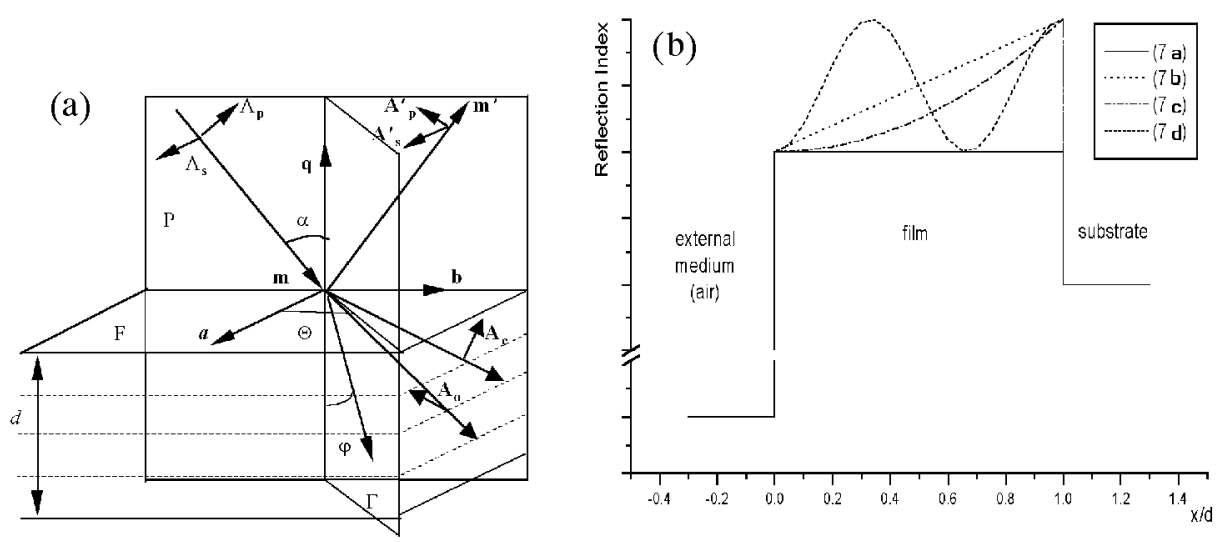

Fig. 1. (a) The reflection of light on the isotropic medium-uniaxial crystal boundary: $\alpha$ - angle of incidence, $\varphi$ - the angle between optical axis $c$ and the normal to the boundary $q, \Theta$ - azimuthal angle measured from the normal to the plane of incidence, $\boldsymbol{m}, \boldsymbol{m}^{\prime}$ - vectors of refraction of incident and reflected waves; $A_{\mathrm{p}, \mathrm{s}}, A_{\mathrm{p}, \mathrm{s}}^{\prime}-$ complex amplitudes of incident and reflected waves, respectively, $\mathrm{p}$ - corresponds to parallel, s to perpendicular component to the plane of incidence, $A_{\circ}, A_{\mathrm{e}}$ - complex amplitudes of past ordinary and extraordinary waves in a uniaxial chip. (b) Lateral views of refractive index of non-homogeneous film, defined by Eqs. (7). The curves (7a)- $(7 \mathrm{~d})$ correspond to a lateral view of refractive index of the anisotropic film.

Thus the basic equation of reflective ellipsometry for the boundary of two media looks like this [2]:

$$
\rho=\tan (\psi) \mathrm{e}^{\mathrm{i} \Delta}=\frac{\rho^{0} R_{\mathrm{pp}}+R_{\mathrm{ps}}}{R_{\mathrm{ss}}+\rho^{0} R_{\mathrm{sp}}},
$$

where $\Delta$ and $\psi$ - ellipsometric angles that are measured experimentally. Here $\rho^{0}=A_{\mathrm{p}} / A_{\mathrm{s}}$ - polarization relation for the incident wave. In experiments one usually takes $A_{\mathrm{p}}=A_{\mathrm{s}}$ and then $\rho^{0}=1$.

The case when the optic axis of the second medium is directed along the normal to the boundary [5] represents the practical concern. In this case the matrices of reflection $R$ and transmission $T$ have a diagonal view $\left(R_{\mathrm{ps}}=R_{\mathrm{sp}}=T_{\mathrm{es}}=\right.$ $T_{\mathrm{op}}=0, R_{\mathrm{pp}}=R_{\mathrm{p}}, R_{\mathrm{ss}}=R_{\mathrm{s}}, T_{\mathrm{ep}}=T_{\mathrm{p}}, T_{\mathrm{os}}=T_{\mathrm{s}}$ ), and the basic equation of ellipsometry at $\rho^{0}=A_{\mathrm{p}} / A_{\mathrm{s}}=1$ will look like

$$
\rho=\tan (\psi) \mathrm{e}^{\mathrm{i} \Delta}=\frac{R_{\mathrm{p}}}{R_{\mathrm{s}}} .
$$


Let us consider single-layer system, in which $N_{10}=N_{1 \mathrm{e}}=N_{1}, N_{2 \circ}(x)$, $N_{2 e}(x), N_{30}, N_{3 e}$ are complex indices of refraction of ordinary (o) and extraordinary (e) waves of environment, film, and substrate, respectively. The indices of refraction of film $N_{20}(x), N_{2 \mathrm{e}}(x)$ depend on the thickness of the explored film (Fig. 1b).

For a homogeneous film $\left(N_{2 \mathrm{o}}(x)=\right.$ const, $N_{2 \mathrm{e}}(x)=$ const $)$ with arbitrary orientation of optical axis along the boundary, the reflectivities and transmissions can be written in a matrix form [2]:

$$
\begin{aligned}
& R=R^{(12)}+T^{(21)} R^{(23)}\left(1+X^{(21)} R^{(21)} X^{(23)} R^{(23)}\right)^{-1} T^{(12)}, \\
& T=T^{(21)} X^{(21)} T^{(23)}\left(1+X^{(21)} R^{(21)} X^{(23)} R^{(23)}\right)^{-1} T^{(12)},
\end{aligned}
$$

where $R^{(12)}, R^{(21)}, R^{(23)}$ — reflection matrices on boundary air-film, film-air, and film-substrate, respectively, $T^{(12)}, T^{(21)}, T^{(23)}$ - transmission matrices on boundary air-film, film-air, and film-substrate, respectively; $X^{(23)}, X^{(21)}$ — phase progression matrices of waves in an anisotropic layer at the motion from boundary air-film to boundary film-substrate and back, respectively.

The case when optical axes of the film and the substrate are oriented along the normal to the boundary represents the practical concern. In this case the expressions for reflectivities and transmissions are considerably simplified [6]:

$$
R_{\mathrm{p}, \mathrm{s}}=\frac{r_{\mathrm{p}, \mathrm{s}}^{(12)}+r_{\mathrm{p}, \mathrm{s}}^{(23)} X_{\mathrm{p}, \mathrm{s}}^{2}}{1+r_{\mathrm{p}, \mathrm{s}}^{(12)} r_{\mathrm{p}, \mathrm{s}}^{(23)} X_{\mathrm{p}, \mathrm{s}}^{2}}, \quad T_{\mathrm{p}, \mathrm{s}}=\frac{\left(1+r_{\mathrm{p}, \mathrm{s}}^{(12)}\right)\left(1+r_{\mathrm{p}, \mathrm{s}}^{(23)}\right) X_{\mathrm{p}, \mathrm{s}}}{1+r_{\mathrm{p}, \mathrm{s}}^{(12)} r_{\mathrm{p}, \mathrm{s}}^{(23)} X_{\mathrm{p}, \mathrm{s}}^{2}},
$$

where $R_{\mathrm{p}, \mathrm{s}}^{(12)}, r_{\mathrm{p}, \mathrm{s}}^{(23)}$ - reflectivities of $\mathrm{p}$ - and s-polarized waves on the boundary air-film and film-substrate, respectively, $X_{\mathrm{p}, \mathrm{s}}=\exp \left(-2 \mathrm{i} \pi \eta_{\mathrm{o}, \mathrm{e}} d / \lambda N_{1}^{1 / 2}\right)-$ phase progression of electromagnetic waves on thickness of the film, $\lambda-$ wavelength in air, $d$ - the thickness of the film.

The reflectivities $r_{\mathrm{p}, \mathrm{s}}^{(i j)}(i, j=1,2,3)$ are equal to

$$
r_{\mathrm{p}}^{(i j)}=\frac{N_{j \circ}^{2} \eta_{\mathrm{e} i}-N_{i \mathrm{o}}^{2} \eta_{\mathrm{ej}}}{N_{j \circ}^{2} \eta_{\mathrm{e} i}+N_{i \mathrm{o}}^{2} \eta_{\mathrm{ej}}}, \quad r_{\mathrm{s}}^{(i j)}=\frac{\eta_{\circ i}-\eta_{\circ j}}{\eta_{\circ i}+\eta_{\circ j}} .
$$

Here $\eta_{\circ i}=\left(N_{\circ i}^{2}-\xi^{2}\right)^{1 / 2}, \eta_{e i}=\left(N_{o i}^{2}-N_{o i}^{2} \xi^{2} / N_{e i}^{2}\right)^{1 / 2}, \xi=N_{1} \cos (\alpha)$ - normal and tangential components of refraction vectors [7] of ordinary and extraordinary waves in the film.

Now we shall transfer to viewing a non-homogeneous uniaxial film, whose optical axis is directed along the normal to the boundary, on an isotropic substrate. Such systems are typical at manufacturing of organic films such as LangmuirBlodgett type [6]. For non-homogeneous film, as follows from expressions (4a), the reflectivity depends on thickness of the film: $R_{\mathrm{p}, \mathrm{s}}=R_{\mathrm{p}, \mathrm{s}}(d)$. Unfortunately, it was impossible to receive analytical expression for reflectivities of non-homogeneous films. Therefore we will use the following operations for finding the reflectivities: let us divide a non-homogeneous layer $d$ into $k$ equal parts so that it is possible 
to consider each of partial layers as homogeneous. Thus on the basis of expressions (4a) we shall make an iterative procedure permitting to find reflectivities of system of $k$ homogeneous layers

$R_{\mathrm{p}, \mathrm{s}}^{(j)}=\frac{r_{\mathrm{p}, \mathrm{s}}^{(j, j+1)}+R_{\mathrm{p}, \mathrm{s}}^{(j+1)}\left(X_{\mathrm{p}, \mathrm{s}}^{(j+1)}\right)^{2}}{1+r_{\mathrm{p}, \mathrm{s}}^{(j, j+1)} R_{\mathrm{p}, \mathrm{s}}^{(j+1)}\left(X_{\mathrm{p}, \mathrm{s}}^{(j+1)}\right)^{2}}, \quad T_{\mathrm{p}, \mathrm{s}}^{(j)}=\frac{\left(1+r_{\mathrm{p}, \mathrm{s}}^{(j, j+1)}\right) T_{\mathrm{p}, \mathrm{s}}^{(j+1)} X_{\mathrm{p}, \mathrm{s}}^{(j+1)}}{1+r_{\mathrm{p}, \mathrm{s}}^{(j, j+1)} R_{\mathrm{p}, \mathrm{s}}^{(j+1)}\left(X_{\mathrm{p}, \mathrm{s}}^{(j+1)}\right)^{2}}$.

Here $j=k-1, k-2 \ldots, 2,1$ is the number of viewed boundary of partial layers. The calculations start for the boundary of the $k$-th partial layer and the substrate. The obtained reflectivity $R_{\mathrm{p}, \mathrm{s}}^{(k-1)}$ at $j=k-1$ corresponds to reflection from the $k$-th partial layer on the substrate, $r_{\mathrm{p}, \mathrm{s}}^{(k-1, k)}$ - reflectivity on the boundary of $(k-1)$-th partial layer and $k$-th partial layer. The obtained reflectivity $R_{\mathrm{p}, \mathrm{s}}^{(k-1)}$ will be used for calculation of reflectivity $R_{\mathrm{p}, \mathrm{s}}^{(k-2)}$ of a system of two partial layers on the substrate, etc. At $j=1$ on the basis of the iterative procedure (6) the reflectivity $R_{\mathrm{p}, \mathrm{s}}=R_{\mathrm{p}, \mathrm{s}}^{(0)}$ of the system of $k$ partial layers, that is reflectivity from non-homogeneous layered system will be calculated. The calculations of transmission $T_{\mathrm{p}, \mathrm{s}}=T_{\mathrm{p}, \mathrm{s}}^{(0)}$ for non-homogeneous system are similar.

The obtained iterative procedure at great values of $k$ allows to find elements of matrices of reflection (1) and with their help on the base of major equation of ellipsometry (2) to define ellipsometric parameters $\Delta$ and $\psi$ of a non-homogeneous anisotropic layer on the substrate. Let us also notice that expressions (4a)-(6) are to be used only for the special case of orientation of optical axis along the normal to the boundary, when matrices of reflection and the transmission are diagonal. In the general case it is necessary to use matrix recursions relations on the basis of expression (4), and to discover reflectivities and transmissions on the boundary of partial layers on the basis of the boundary problem solution for anisotropic media $[7]$.

\section{Numerical modelling of a non-homogeneous anisotropic layer}

While examining anisotropic non-homogeneous films we were interested in the influence of three factors on $\Delta-\psi$ diagrams:

a) type of the inhomogeneity of the film;

b) absorbance of the film;

c) degree of anisotropy of the film.

It was shown for isotropic non-homogeneous transparent films [8] that the linear gradient of refractive index essentially influences $\Delta-\psi$ diagrams, constructed depending on the thickness of the film.

Let us use the iterative procedure (6) for the modelling of dependence of ellipsometric angles $\Delta$ and $\psi$ at reflecting of light on the non-homogeneous film, whose optical axis is directed along the normal to the boundary. Let us consider the following systems: a $\mathrm{ZnO}$ film on the substrate of melted quartz $\mathrm{SiO}_{2}$, and also a film of Langmuir-Blodgett's type (LB) on the substrate of melted quartz $\mathrm{SiO}_{2}$, which are widely applied in integrated optics and acoustoelectronics. 
It was supposed that the measurements are carried out at a wavelength $\lambda=$ $632.8 \mathrm{~nm}$ for films, whose thickness $d$ is ranged between $0-300 \mathrm{~nm}$. At modelling the number of dissections reached $k=50$ with the thickness of a partial layer $6 \mathrm{~nm}$. As an environment we took air $\left(N_{1}=N_{10}=N_{1 \mathrm{e}}=(1.000,0.000)\right)$, and as a substrate - melted quartz $\left(N_{30}=N_{3 e}=(1.460,0.000)\right)$. The change of the film refractive indices depending on the thickness was taken not greater than $3 \%$. Let us suppose that during cultivation of the film, refractive indices on the boundary with the substrate have values $N_{20}^{(2)}, N_{2 \mathrm{e}}^{(2)}$, and on the boundary with the surrounding medium $N_{2 o}^{(1)}, N_{2 e}^{(1)}$. Then on the air-film boundary for $\mathrm{ZnO}$ we took the values $N_{20}^{(1)}=(1.990,0.000), N_{2 \mathrm{e}}^{(1)}=(2.067,0.000)$, and $N_{20}^{(1)}=(1.470,0.000)$, $N_{2 \mathrm{e}}^{(1)}=(1.490,0.000)$ [9] for the Langmuir-Blodgett's film. On the film-substrate boundary for $\mathrm{ZnO}$ we took the values $N_{20}^{(2)}=(2.050,0.000), N_{2 \mathrm{e}}^{(2)}=(2.129,0.000)$, and for the Langmuir-Blodgett's film $N_{2 \mathrm{o}}^{(2)}=(1.514,0.000), N_{2 \mathrm{e}}^{(2)}=(1.524,0.000)$ were taken. The imaginary part in a complex refractive index corresponded to an absorption index.

\subsection{Modelling for different types of non-homogeneity of the film}

Let us set the laws of changing of the film refractive indices in the following way (Fig. 1b):

$$
\begin{aligned}
& N_{2 i}(x)=N_{2 i}^{(1)}=\mathrm{const}, \quad i=(\mathrm{e}, \mathrm{o}), \\
& N_{2 i}(x)=N_{2 i}^{(1)}+\left(N_{2 i}^{(2)}-N_{2 i}^{(1)}\right) x / d, \quad i=(\mathrm{e}, \mathrm{o}), \\
& N_{2 i}(x)=N_{2 i}^{(1)}+\left(N_{2 i}^{(2)}-N_{2 i}^{(1)}\right)(x / d)^{2}, \quad i=(\mathrm{e}, \mathrm{o}), \\
& N_{2 i}(x)=0.5\left[\left(N_{2 i}^{(1)}+N_{2 i}^{(2)}\right)-\left(N_{2 i}^{(2)}-N_{2 i}^{(1)}\right) \cos (1.5 \pi x / d)\right], i=(\mathrm{e}, \mathrm{o}) .
\end{aligned}
$$

Here $x$ is the distance from the border of the film to the surrounding medium, $0 \leq x \leq d$.

The expression (7a) corresponds to a homogeneous film, (7b) - to the linear increase in indices of refraction of the film from $N_{2 i}^{(1)}$ up to $N_{2 i}^{(2)},(7 \mathrm{c})$ - quadratic, (7d) - periodical change on thickness of the film.

The results of calculations of ellipsometric quantities at the angle of incidence $\alpha=50^{\circ}$ are given in Fig. 2: (a) for a $\mathrm{ZnO}$ film, (b) for an LB film. The numerical modelling shows that for films with the indices of refraction considerably different from the substrate refractive index (Fig. 2a), it is impossible to reveal the type of non-homogeneity of the film - the curves of different types of gradient are close to each other. Let us also notice that for anisotropic films varying from isotropic $\left(N_{20}=N_{2 \mathrm{e}}\right) \Delta-\psi$ diagrams have spiral view with self-crossings, i.e. that with increase in thickness of the film there is no recurrence of ellipsometric angles depending on the thickness of the film. 

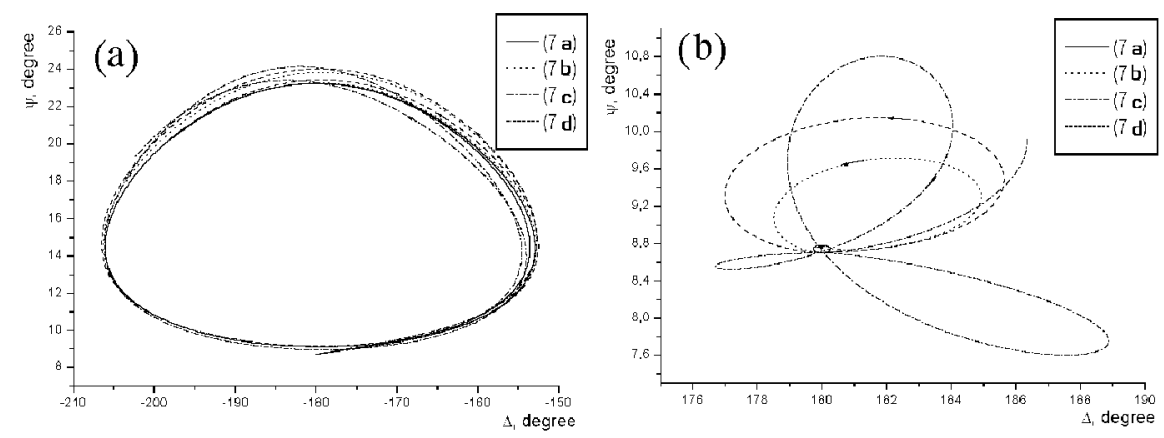

Fig. 2. Dependences of ellipsometric angles $\Delta$ and $\psi$ on the width $d$ of the film $(0-300 \mathrm{~nm})$. Angle of incidence $\alpha=50^{\circ}$. The curves calculated with Eqs. (7a) $-(7 \mathrm{~d})$ correspond to a lateral view of refractive index of the anisotropic film: (a) $\mathrm{ZnO}$ film; (b) LB film.

For films with indices of refraction close to the index of refraction of the substrate, the change of film refractive index caused by thickness change leads to considerable changes of ellipsometric angles. Figure $2 \mathrm{~b}$ shows that for a homogeneous film the changes of angles $\Delta$ and $\psi$ are negligible (curve 1), while for films with lapse rates of refractive indices they can make up to ten degrees. Thus for films with periodically varying indices of refraction, spirals with self-crossings are brightly expressed. The spiral dependence is characteristic of immersing film systems [5], but a self-crossing of curves is possible only if there is a lapse rate of index of refraction. The calculations show that for films with indices of refraction close to index of refraction of the substrate, the differences of ellipsometric angles on $\Delta-\psi$ diagrams, which can be experimentally measured, start to be visible from thicknesses of approximately 10-20 nm. It allows to develop solutions of inverse problems of ellipsometry for non-homogeneous layers and to define the type of the lapse rate of the film refractive index.

It is evident that the largest information about the quantity of a non-homogeneity of the film can be obtained at conditions, when the influence of the substrate on ellipsometric angles is reduced to minimum. Therefore the greatest information about the film can be obtained at angles of incidence close to the Brewster angle. If the substrate represents a uniaxial chip with indices of refraction $N_{30}, N_{3 \mathrm{e}}$ and with optical axis oriented along the normal to the boundary, then the Brewster angle for the uniaxial chip-uniaxial chip boundary is defined by the formula

$$
\sin \left(\alpha_{\mathrm{Br}}\right)=\frac{N_{\mathrm{e} 2} N_{\mathrm{e} 3}}{N_{\mathrm{o} 2}} \sqrt{\frac{N_{\mathrm{o} 3}^{2}-N_{\mathrm{o} 2}^{2}}{N_{\mathrm{o} 3}^{2} N_{\mathrm{e} 3}^{2}-N_{\mathrm{o} 2}^{2} N_{\mathrm{e} 2}^{2}}} .
$$

For an air-isotropic substrate system we easily gain the known expression

$$
\sin \left(\alpha_{\mathrm{Br}}\right)=\frac{N_{3}}{\sqrt{1+N_{3}^{2}}},
$$


from which we find $\alpha_{\mathrm{Br}}=55^{\circ}, 95$ for the air-melted quartz system.

Now we take films with indices of refraction close to the index of refraction of a substrate. As can be seen from Fig. 3, in such cases the responsiveness of measurements of ellipsometric parameters is the largest for angles close to the Brewster angle. For angles greater than $\alpha_{\mathrm{Br}}$, the reflectivity $R_{\mathrm{p}}$ changes the phase into $180^{\circ}$, therefore curves on the diagram are of different type, transferring from closed to unclosed.
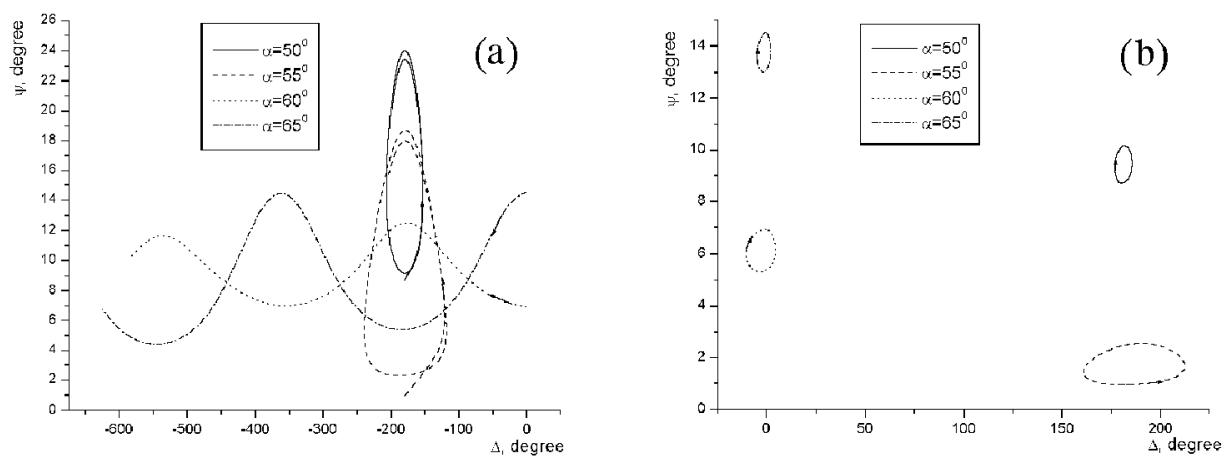

Fig. 3. Dependences of ellipsometric angles $\Delta$ and $\psi$ on the width $d$ of the film (0-300 nm) for a linear change of refractive index at different angles of incidence: (a) $\mathrm{ZnO}$ film; (b) LB film.

\subsection{Modelling for different uptake degree of the film and for a different degree of the film anisotropy}

Further we will examine the films such as LB type, with refractions close to refraction of the substrate. We will be restricted to modelling of layered systems with a linear dependence of refractive index of ( $7 \mathrm{~b})$ type and we will consider the influence of absorption and anisotropy of the film on ellipsometric angles $\Delta$ and $\psi$. The results of modelling, depending on an uptake of the film are represented in Figs. 4a, b and the ones depending on the degree of the film anisotropy - in Fig. 5a, b.

The absorption to occurrence of spiral dependences, and the tendency of increasing the total amplitude of the spiral on $\Delta-\psi$ diagram with increase in absorption can be observed. At small absorption the curves are nearly closed curves, but with increase in the coefficient of absorption the spiral-like dependences with self-crossing occur. A self-crossing is an idiosyncrasy of uniaxial non-homogeneous films. For angles of incidence greater than the Brewster angle ( $\alpha=60^{\circ}$, Fig. $\left.4 \mathrm{a}\right)$ the sizes of the spiral will increase monotonically. The absorption essentially influences the dependences at an absorption coefficient $>0.01$. Thus the position and the total amplitude of the curve for an immersing film differs from a transparent 

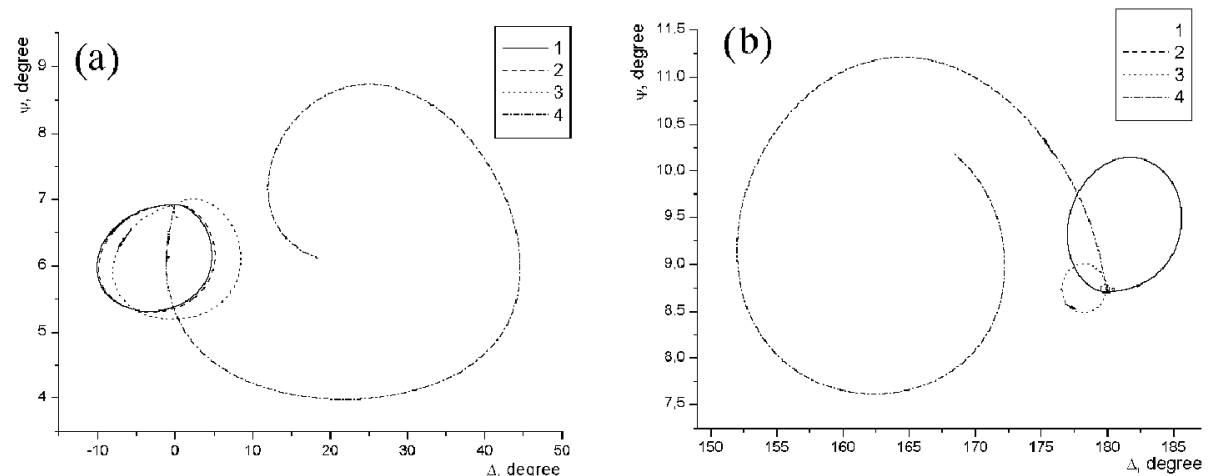

Fig. 4. Dependences of ellipsometric angles $\Delta$ and $\psi$ on the width $d$ of the film $(0-300 \mathrm{~nm})$ for linear change of refractive index of an LB film at different coefficients of absorption of the film. Curves: $1-\operatorname{Im}\left(N_{20}\right)=\operatorname{Im}\left(N_{2 \mathrm{e}}\right)=$ $0.000 ; 2-\operatorname{Im}\left(N_{20}\right)=\operatorname{Im}\left(N_{2 \mathrm{e}}\right)=0.001 ; 3-\operatorname{Im}\left(N_{20}\right)=\operatorname{Im}\left(N_{2 \mathrm{e}}\right)=0.010 ; 4-$ $\operatorname{Im}\left(N_{20}\right)=\operatorname{Im}\left(N_{2 \mathrm{e}}\right)=0.100$. Angles of incidence: (a) $\alpha=60^{\circ}$, (b) $\alpha=50^{\circ}$.
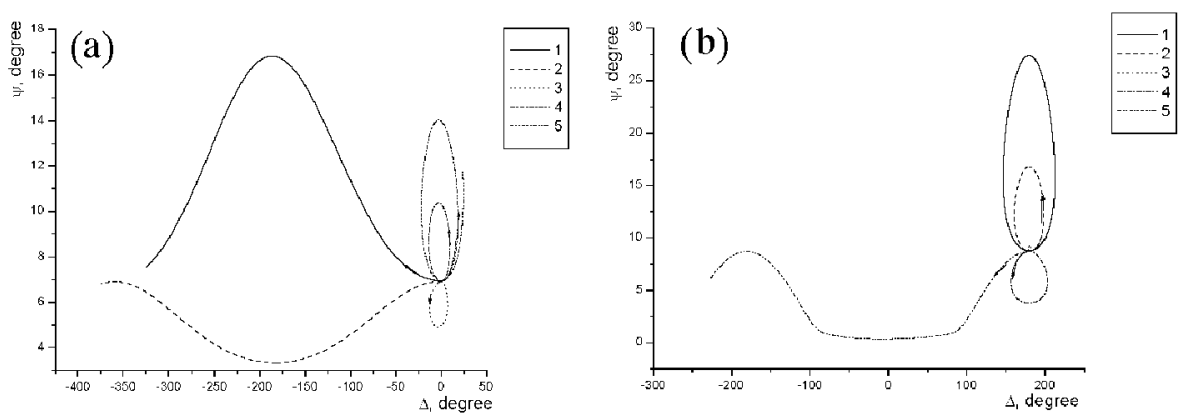

Fig. 5. Dependences of ellipsometric angles $\Delta$ and $\psi$ on the width $d$ of the film $(0-300 \mathrm{~nm})$ for linear change of index of refraction of an LB film at different coefficients of an uptake of the film. Curves: $1-\frac{N_{e}-N_{0}}{N_{0}}=-20 \% ; 2-\frac{N_{e}-N_{0}}{N_{0}}=-10 \%$; $3-\frac{N_{e}-N_{0}}{N_{0}}=0 \% ; 4-\frac{N_{e}-N_{0}}{N_{0}}=10 \% ; 5-\frac{N_{e}-N_{0}}{N_{0}}=20 \%$. Angles of incidence: (a) $\alpha=60^{\circ}$, (b) $\alpha=50^{\circ}$.

film on quantities, which can be experimentally measured. However for angles of incidence smaller than the Brewster angle $\alpha_{\mathrm{Br}}\left(\alpha=50^{\circ}\right.$, Fig. 4b) the anomaly appears. First, the total amplitude of the curve on the diagram decreases, and then it increases. This behaviour of the curve can be explained by competition of anisotropy and absorption. The existence of the anisotropy of the film cancels the increase in ellipsometric angles at particular quantities of absorption. Second, the absorption starts to predominate and influences the view of the diagrams more essentially. It means that at an experimental research of non-homogeneous 
anisotropic immersing films the uptake in the film will be seen first of all.

In Figs. 5a, b the dependences of ellipsometric angles for different coefficients of anisotropy of the transparent film such as LB for the linear lapse rate of refractive index $(7 \mathrm{~b})$ are represented. The coefficient of anisotropy of the film

$$
\frac{\Delta N}{N_{\circ}}=\frac{N_{\mathrm{e}}-N_{\mathrm{o}}}{N_{\mathrm{o}}}
$$

varied from $-20 \%$ (optically negative uniaxial film) up to $+20 \%$ (optically positive uniaxial film). Thus everywhere in calculations the ordinary index of refraction on air-film boundary $N_{20}^{(1)}=(1.470,0.000)$ was taken, and on film-substrate boundary $N_{20}^{(2)}=(1.514,0.000)$, i.e. that the coefficient of the lapse rate was $3 \%$. For an angle of incidence $\alpha=60^{\circ}$ (Fig. 5a) with the change of anisotropy from $-20 \%$ up to $+20 \%$ the transition from unlocked curves to closed happens, and also changes the protuberance of the curves. The view of curves is similar to calculated in [8] for different coefficients of the film gradient, however in this case the reason of change of view of curves on $\Delta-\psi$ diagram is anisotropy. For an angle of incidence smaller than the Brewster angle ( $\alpha=50^{\circ}$, Fig. 5b) for optically negative films the closed curves can be observed. With the decrease in anisotropy the total amplitude of curves decreases, and at

$$
\frac{\Delta N}{N_{\circ}}=\frac{N_{\mathrm{e}}-N_{\mathrm{o}}}{N_{\circ}}=0
$$

it does not exceed $0.5^{\circ}$. For optically positive films an increase in anisotropy leads to a transition from closed to unlocked curves in $\Delta-\psi$ diagram. Thus, the anisotropy of the film makes a great contribution to elipsometric parameters, similar to contribution of the gradient of the film refractive index. Hence, in order to differentiate between the anisotropic and isotropic contributions to the composite character of the diagrams, which are obtained in course of ellipsometric investigations of the films structures, during the process of their growth, the additional investigations are required.

\section{Conclusion}

On the base of the obtained iterative formula for calculation of reflectivities from system "a non-homogeneous uniaxial film on a uniaxial substrate with optical axis, directed along the normal to the boundary" and numerical modelling of system it is possible to make the following conclusions:

- the non-homogeneity of the film is in the best way shown for refractive index close to that of the substrate;

- the type of non-homogeneity of the film with low refractive index gradients can be determined for thicknesses greater than $20 \mathrm{~nm}$ by the form of a curve on $\Delta-\psi$ diagram; 
- the measurements of ellipsometric parameters are expected to be conducted at angles of incidence, close to the Brewster angle for the air-substrate system;

- the influence of uptake of anisotropic films on ellipsometric angles leads to spiral dependences with self-crossings;

- the influence of the coefficient of anisotropy for films with low gradients of index of refraction on elipsometric angles is similar to influence of the large gradient of index of refraction for isotropic films.

\section{References}

[1] Thin Films - Interdiffusion and Reactions, Eds. J.M. Poate, K.N. Tu, J.W. Mayer, Wiley, New York 1978, p. 576.

[2] R.M.A. Azzam, N.M. Bashara, Ellipsometry and Polarized Light, North-Holland, Amsterdam 1977, p. 583.

[3] The Fundamentals of Ellipsometry, Ed. A.W. Rzhanov, Nauka, Novosibirsk 1979, p. 419.

[4] A. Yariv, P. Yeh, Optical Waves in Crystals, Wiley, New York 1984, p. 616.

[5] V.V. Filippov, A.Yu. Tronin, A.F. Konstantinova, Crystallography 39, 360 (1994).

[6] A.Y. Tronin, A.F. Konstantinova, V.V. Filippov, M.M. Karpuk, Proc. SPIE 1270, 151 (1990).

[7] L.M. Brekhovskikh, Waves in Stratified Mediums, Nauka, Moscow 1973, p. 343.

[8] F.I. Fedorov, Optics of Anisotropic Media, Belarus Academy Sciences Publishers, Minsk 1958, p. 380.

[9] G.K. Carniglia, J. Opt. Soc. Am. A 7, 848 (1990).

[10] Acoustic Crystals, Ed. M.P. Shaskolskaya, Nauka, Moscow 1982, p. 632. 